apportioned telephone review to discuss Results and/or symptoms. As per NICE guidance annual Fibroscan was organised through a dedicated clinic. If any clinical and/or biochemical concerns arose a face-to-face review in clinic is arranged.

Results Over this period 45 patients received 6-monthly virtual HBV clinic review. Bloods showed a median peak ALT $36 \mathrm{u} / \mathrm{L}$ $(0-41 \mathrm{u} / \mathrm{L})$, median viral load $62 \mathrm{iu} / \mathrm{ml}$. and median AFP 2.7. Only $3 / 45$ required face-to-face follow up due to a possible flare, with $2 / 45$ eventually requiring an antiviral agent (Tenofovir). No patients required admission and/or access to other acute and/or community services due to their liver disease during this period. The cost savings were considerable. 6 monthly reviews in a face-to-face clinic over the 2.5 year period would have cost an estimated $£ 27636$. The cost of running a virtual telephone clinic for the same period was $£ 5,517$; a saving of $501 \%$. There were only 5 missed blood tests ahead of the clinic appointment. This compares to the $22 \%$ DNA rate in our general hepatology clinics.

Conclusions A nurse-led virtual clinic provides high-quality, cost-effective care to patients and improves HBV monitoring. Better compliance allows for detection of flares which could require antiviral treatment. With the prospect of new treatments on the horizon it is important these patients remain under the care of a hepatology team and a virtual clinic model allows them to remain engaged with Liver services.

\section{PTH-087 IMPROVING HEPATITIS C DETECTION AND TREATMENT VIA COMMUNITY-BASED SERVICES}

Douglas Corrigall*, Linda Porter, Sarah Fairclough, Katrina White, Denise Killworth, Gavin Wright. Basildon And Thurrock University Hospital, Basildon, UK

\subsection{6/gutjnl-2018-BSGAbstracts.243}

Introduction With the advent of highly efficacious Direct Acting Antiviral (DAA) Hepatitis C (HCV) therapy, the World Health Organisation project global HCV eradication by 2030 and NHS-England is targeting HCV eradication nationally by 2025. Despite a nationwide HCV operational delivery network it is estimated that $\sim 90 \%$ of those infected in England are not actively engaged with secondary/tertiary HCV services providing therapy and many, often asymptomatic, remain untested and undiagnosed. These patients do engage with primary care; but with increasing pressures on these services and absence of national screening, detection and onward referral to treatment centres are poor. This is a barrier to achieving this target, and the new frontier for HCV services is improved detection and engagement in the community. People with a history of intravenous drug and alcohol misuse have a high prevalence of $\mathrm{HCV}$, are often sexually active with higher rates of transmission, have limited access to and/or engagement with HCV services, but often do attend community drug and alcohol services (CDAS). Our aims were to increase identification and treatment of patients with HCV by engaging these individuals within a community-based setting.

Methods Over one year (August 2015-2016), in partnership with five local CDAS we provided onsite nurse-led consultation, counselling, screening and risk stratification through noninvasive measurement of liver stiffness (fibroscan), dried blood spot screening (HBV/HCV/HIV serology, HCV RNA, T-spot), and referral to secondary care for initiation of approved DAA therapy and ongoing management of any concomitant chronic liver disease.
Results 174 CDAS service-users were screened and 123 (70\%) were diagnosed as HCV RNA positive; 54\% Genotype 3\% and $46 \%$ Genotype 1. Median fibroscan score 7.1 Kpa, with $21(12 \%)$ had a fibroscan Result suggestive of cirrhosis and were prioritised to treatment according to National guidance via our NHS-England HCV ODN.

To-date $86(70 \%)$ pf the HCV positive patients have attended our clinic for consideration of access to DAA therapy.

Conclusions This community-based pilot had a significant rate of detection (70\%), and excellent conversion to secondary care clinic review (70\%). However, the majority of our patients had low levels of fibrosis and as NHS England policy over that period prioritised for patients with advanced disease, this cohort did not receive immediate access to treatment from the ODN over the time of the project, with Resultant disengagement by many from secondary care. Given recent changes in treatment access prioritisation we are now actively reengaging this group which represents an ongoing challenge.

\section{PTH-088 'MORE IS LESS' - PRESENTING WITH ACUTE VARICEAL BLEEDING}

Benjamin Crooks*, Helen White, Vivek Goodoory, Thomas Butler, Mark Murgatroyd, Javaid Iqbal. Wythenshawe Hospital, Manchester University Foundation Trust, Manchester, UK

\subsection{6/gutjnl-2018-BSGAbstracts.244}

Introduction Acute variceal bleeding (AVB) has historically accounted for up to $10 \%$ of all GI bleeds necessitating emergency out of hours endoscopy. These patients have a significantly poorer prognosis and higher re-bleed rate than nonvariceal GI haemorrhage. In the last 3 years we have anecdotally noticed a significant reduction in AVB necessitating emergency endoscopy. This has coincided with the employment of dedicated hepatologists. We sought to qualify and quantify this reduction with the employment of one and then a second hepatologist at a university teaching hospital 18 months apart. Methods This was a retrospective review identifying all AVB patients who underwent emergency endoscopy over a 3 year period between January 2015 and December 2017. Data was collected from the electronic database and the GI reporting tool. This included endoscopic findings, therapy performed and whether there was a previous history of AVB requiring endoscopy. A dedicated hepatologist was employed in January 2015 (period 1; 18 months) and a second hepatologist June 2016 (period 2; 18 months).

Results Prior to a dedicated hepatologist all patients were followed up by general gastroenterologists. There was no dedicated variceal banding programme. There were up to 300 acute GI bleed endoscopies a year with approximately $10 \%$ due to AVB. Both hepatologists began performing weekly dedicated oesophageal variceal screening and treatment endoscopy lists (between 1-2/week). During period 1, there were $30 \mathrm{AVB} ; 27 / 30$ (90\%) received therapy, in the remaining 3, banding could not be applied due to poor views and injection therapy or sengstaken tube placement was performed. Of those presenting; 21/30 (70\%) had previous OGD and banding but only $8 / 21(38 \%)$ had previously been on banding program.

During period 2, there were 20 AVB; $19 / 20$ (95\%) received therapy. $12 / 20(60 \%)$ had previous OGD and banding, and only $3 / 20(15 \%)$ were on a dedicated banding programme. 